Supporting Information for

\title{
Large Magnetoresistance at Room Temperature in Organic Molecular Tunnel Junctions With Non-Magnetic Electrodes
}

\author{
Zuoti Xie, ${ }^{1 \dagger}$ Sha Shi, ${ }^{2 \dagger}$ Feilong Liu, ${ }^{2}$ Darryl L. Smith, ${ }^{2}$ P. Paul Ruden,${ }^{2 *}$ and C. Daniel Frisbie ${ }^{1^{*}}$ \\ ${ }^{1}$ Department of Chemical Engineering and Materials Science and ${ }^{2}$ Department of Electrical and Computer \\ Engineering, University of Minnesota, Minneapolis, Minnesota 55455
}

$\uparrow$ Equal contribution

*To whom correspondence should be addressed

P. Paul Ruden: ruden@umn.edu, C. Daniel Frisbie: frisbie@umn.edu 

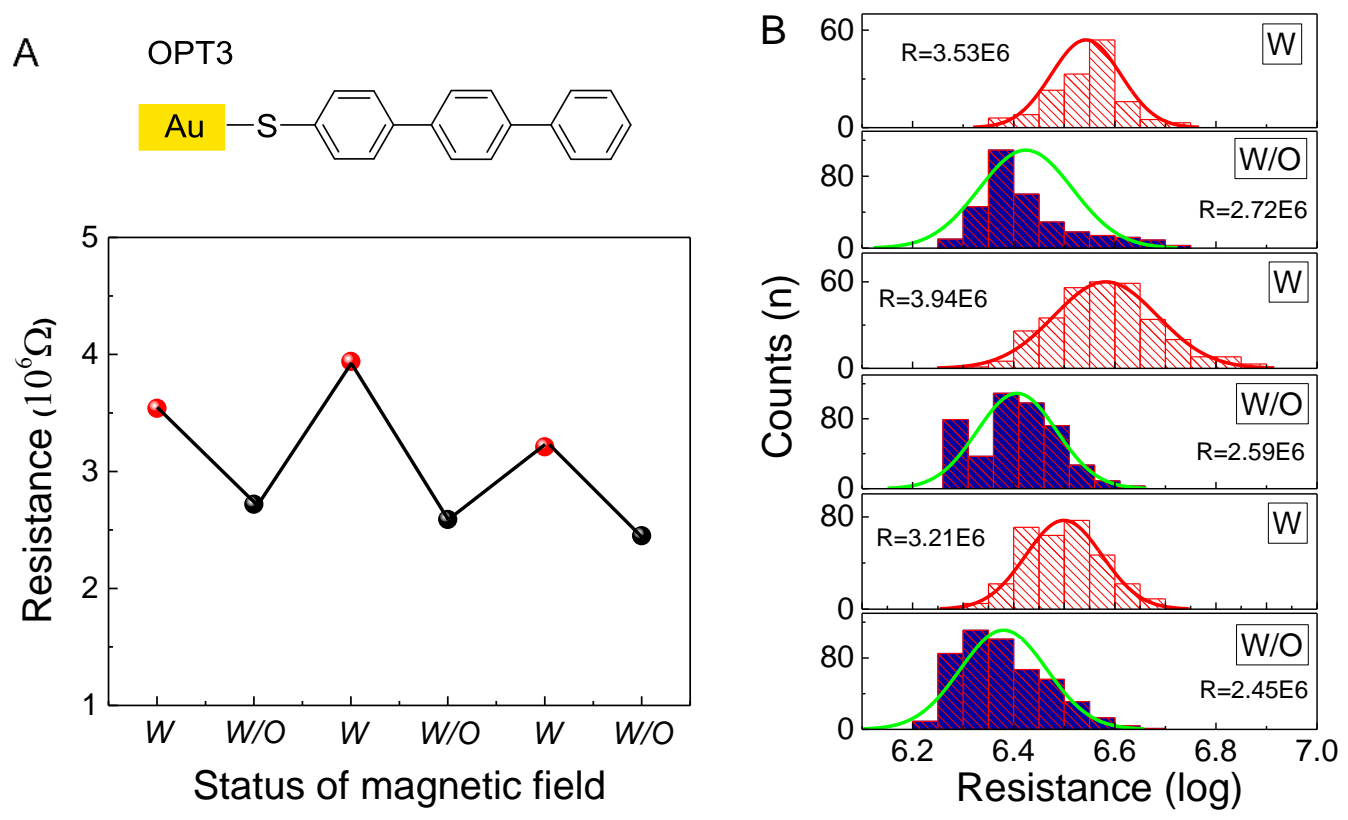

Figure S1. (A) The average low bias resistance with or without the magnetic field perpendicular to the substrate. (B) The corresponding histograms of the measurements.

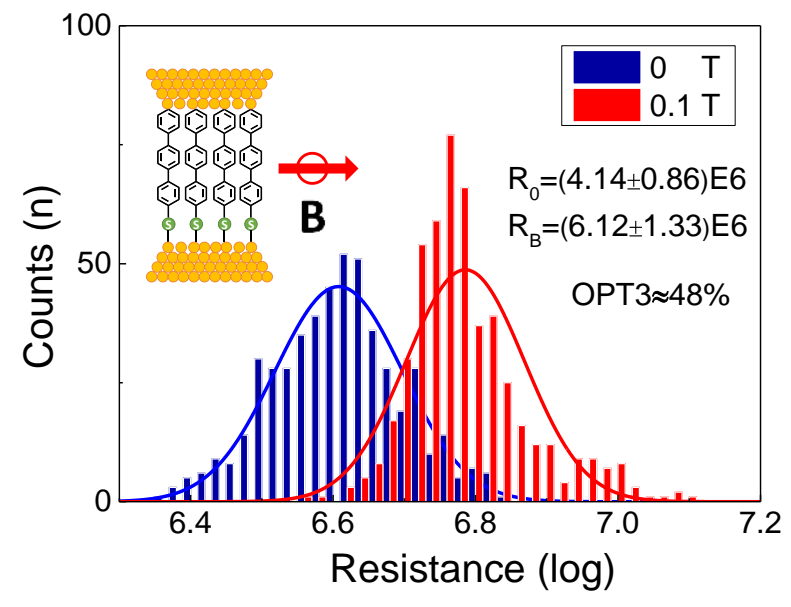

Figure S2. The representative histogram of the low bias resistance of OPT3 junction without (dark blue) or with (red) $50 \mathrm{mT}$ magnetic field parallel to the substrate. 

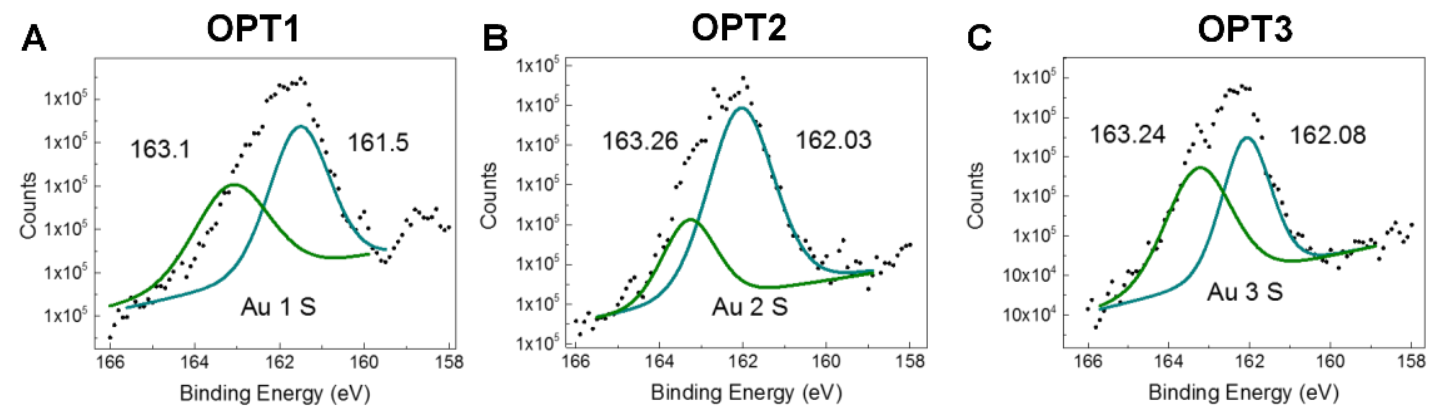

Figure S3. High-resolution XPS spectra of S 2 p core levels of OPTn. $(n=1,2,3)$

\section{Surface potential image}
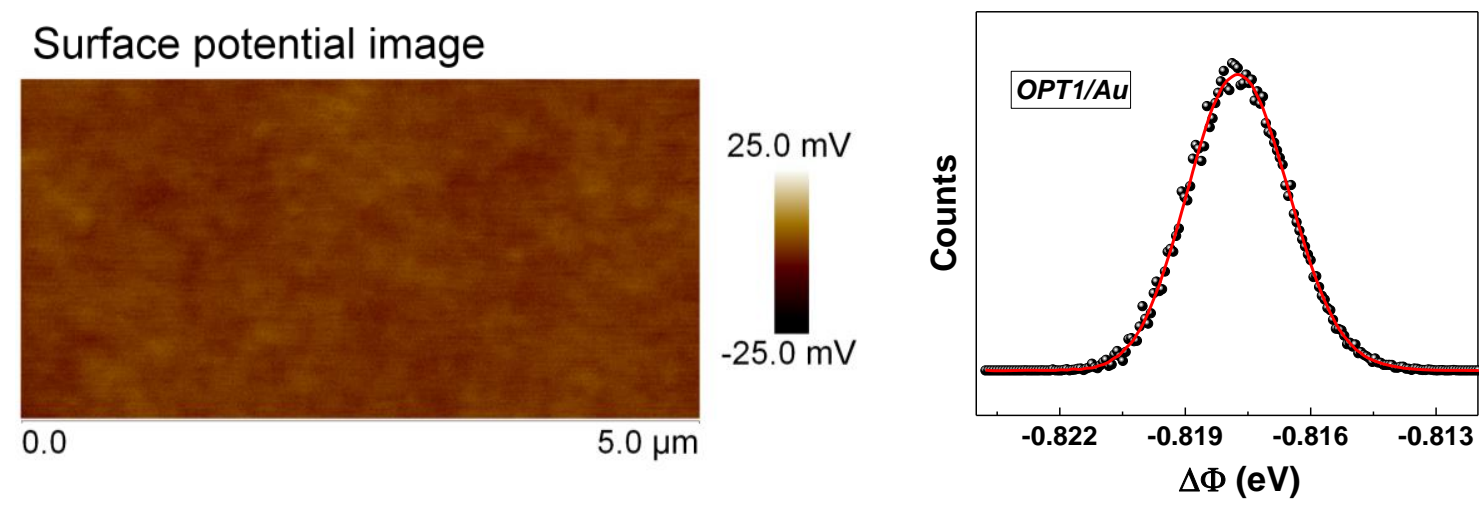

Figure S4. Surface potential image of OPT1 on Au surface and the histogram. 